\title{
Familial Chylomicronemia Syndrome Presenting With Acute Necrotizing Pancreatitis in a Five Month Infant
}

\author{
Borghei $\mathrm{A}^{1}$, Azizi $\mathbf{M}^{2}$
}

${ }^{1}$ Dr. Amirmasoud Borghei, Resident in Paediatrics, Department of Paediatrics, Imam Reza Hospital, Kermanshah University of Medical Sciences, Kermanshah, Iran, ${ }^{2} D r$. Mahba Azizi, MD, Department of Paediatrics, Imam Reza Hospital, Kermanshah University of Medical Sciences, Kermanshah, Iran

Address for Correspondence: Dr. Amirmasoud Borghei, E-mail:amirmasoud56@gmail.com

\begin{abstract}
Familial chylomicronemia syndrome (FCS) is a rare disease characterized by severe fasting hypertriglyceridemia and chylomicronemia, which is inherited in an autosomal recessive manner. It is arisen from apolipoprotein C-II deficiency or Lipoprotein Lipase(LPL) Deficiency.We report a 5-month-old male infant FCS presenting with acute abdominal pain and post surgical diagnosis of acute necrotizing pancreatitis.
\end{abstract}

Key words: Pancreatitis, chylomicronemia, hyperlipidemia, lipoprotein lipase.

\section{Introduction}

$F^{2}$ amilial chylomicronemia syndrome is a genetic defect similar to familial hypercholestrolemia, which influences cleaning of lipoproteins containing apo-B. LPL deficiency or lack of LPL (or its cofactor, apo c-II), facilitates lypolysis and causes a striking rise of triglyceride level (found in serum) in the form of chylomicron, reducing HDL cholesterol level. Contrary to apo c-Il deficiency or lack of apo c-II, chylomicron can be created in the case of LPL deficiency, leading to less increase in triglyceride of serum. Both defects are inherited in an autosomal recessive manner and their incidence is 1 out of $10,00,000^{1}$. The disease usually appears in infancy with episodes of acute pancreatitis. Eruptive xanthoma may occur over the knees, arms and buttocks, and the patients may have hepatosplenomegaly ${ }^{2}$. Here, we describe a 5 month-old male infant with FCS presenting with acute abdomen due to severe necrotizing pancreatitis.

\section{Case Report}

The patient, a 5-month-old male infant, was admitted in Imam Reza Hospital, Kermanshah, Iran, for fever and restlessness. He was the first child in the family, and was born by a normal vaginal delivery; his parents were related (female cousin-male cousin). There was however no record of specific diseases in both the fanilies. There was no history of use of any medicine, or allergies to any medicines or materials. The patient reported to have fever and restlessness since two days before transfer to our hospital. There was only one record of bilious vomiting that had occurred one day before his transfer.

Physical examination showed the patient was feverish and restless. His vital signs included Blood Pressure: 90mmHg (systolic), Respiratory Rate: 32 per minute, Pulse Rate: 124 per minute, Temperatrue: $37.8^{\circ} \mathrm{C}$. Furthermore, his weight and length were $8 \mathrm{~kg}$ and $71 \mathrm{~cm}$ respectively and his head circumference was $46 \mathrm{~cm}$. There was no apparent anomaly. Physical examination revealed abdominal tenderness and possibility of ascites.

He was restless, and there was mild tenderness in epigasteric region. The liver and spleen sizes were in normal range for age.There were no fatty deposits under the skin, and examination of other organs was unremarkable; Fundoscopy was normal. 
Table 1: Showing the Laboratory Investigation Results

\begin{tabular}{|l|l|l|l|}
\hline WBC: $19,100 / \mathrm{mm}^{3}$ & Netrophils: $65 \%$ & Lymphocytes: $33 \%$ & Eosinophils: $2 \%$ \\
\hline MCV: $74.5 \mathrm{fL}$ & Platelets: $3,87,000 / \mathrm{mm}^{3}$ & Haematocrit: $31.9 \%$ & PO $_{4}: 7.0 \mathrm{mg} \%$ \\
\hline Creatinine: $0.5 \mathrm{mg} \%$ & Urea: $20 \mathrm{mg} \%$ & Ca: $8.1 \mathrm{mg} \%$ & \\
\hline Na: $139 \mathrm{meq} / \mathrm{L}$ & K: $5 \mathrm{meq} / \mathrm{L}$ & Bld Sugar: $80 \mathrm{mg} \%$ & AST: $15 \mathrm{IU} / \mathrm{L}$ \\
\hline PT/PTTK: Normal & Alk Phos: $341 \mathrm{IU} / \mathrm{L}$ & ALT: $10 \mathrm{IU} / \mathrm{L}$ & \multicolumn{2}{|l|}{} \\
\hline Triglyceride: $975 \mathrm{mg} \%$ & Cholesterol: $189 \mathrm{mg} \%$ & Amylase: 17 IU/L (5-195 IU/L) \\
\hline Lipase: $138 \mathrm{IU} / \mathrm{L}$ (5-60 IU/L) & U/A \& U/C: Negative. \\
\hline Blood Culture: No Growth. & \multicolumn{2}{l}{}
\end{tabular}

Table 2: Showing laboratory Results after re-admission

\begin{tabular}{|c|c|c|c|}
\hline WBC: $11,500 / \mathrm{mm}^{3}$ & Netrophils: $35 \%$ & Lymphocytes: $60 \%$ & Eosinophils: $5 \%$ \\
\hline MCV: 75fL & Platelets: $2,72,000 / \mathrm{mm}^{3}$ & \multicolumn{2}{|l|}{ ESR:2 } \\
\hline ALT: $10 \mathrm{IU} / \mathrm{L}$ & Alk Phos: $341 \mathrm{IU} / \mathrm{L}$ & Ca: 8 mg\% & $\mathrm{PO}_{4}: 6.9 \mathrm{mg} \%$ \\
\hline AST: 12 IU/L & CPK: 107 IU/L & \multicolumn{2}{|l|}{ ABG: Normal } \\
\hline \multicolumn{2}{|c|}{ Lipase: 103 IU/L (5-60 IU/L) } & \multicolumn{2}{|c|}{ Amylase: 10 IU/L (5-195 IU/L) } \\
\hline \multicolumn{2}{|c|}{ Triglyceride: 2348 mg\% } & \multicolumn{2}{|c|}{ Cholesterol: 479 mg\% } \\
\hline
\end{tabular}

Abdominal x-ray revealed no significant finding except probability of a ureteral stone.Examination of ascites fluid showed Glucose: $253 \mathrm{mg} / \%$, protein $=6.3$ $\mathrm{mg} / \mathrm{dl}$ and culture was negative.

In the patient's initial abdominal ultra-sonogram, a considerable amount of ascites was reported. Considering the possibility of urinary stone in the right ureter and a suspicion of urinoma, an IVP was performed for the patient but it was reported as normal. The patient was reffered for second abdominal ultra-sonogram that showed free liquid containing septa in abdominal cavity and pelvis. Since the intestinal peristaltis had reduced; peritonitis was suspected so a laparotomy was performed.

Surgical exploration showed abundant exudative effusions and fibrins, along with inflammation of the pancreas and there was edema also. According to the surgical findings a diagnosis of acute pancreatitis was made. Allmeasures required for treating peritonitis was made, and after a week, he was discharged as a cured and normal patient.

Two weeks later, the patient was referred back, because he again had abdominal pain and restlessness. In the initial examination, the abdomen was soft but there was mild tenderness. The cardiovascular and respiratory systems were normal and he did not have fever. There were no specific deposits under the skin and other organs were normal on examination.

The blood sample, meanwhile, was completely milky (chylomicron). Considering the tests results and the disease symptoms, familial chylomicronemia syndrome (Type I hyperlipidemia) was suspected. After giving Fresh Frozen Plasma (FFP) to the patient (which was prescribed for acute stage of his disease), symptoms and triglyceride levels reduced.The patient, meanwhile, was being treated with fat-restricted diet along with fat-soluble vitamins. Medium-chain fats were recommended to be consumed.

\section{Discussion}

(FCS) is a rare disease which is inherited in an autosomal recessive manner. It is characterized with severe fasting hypertriglyceridemia and chylomicron plasma, which arises from apolipoprotein C-II or LPL deficiency. In addition to familial hypertriglyceridemia, chylomicronemia also appears along with other diseases including diabetes mellitus, alcohol consumption, estrogen and glucosteroid intake and uremia. Familial LPL deficiency is the most prevalent cause for molecule defect leading to FCS, with an incidence of $1 / 1000000^{1}$.

It may usually appear in the early infancy or adolescence with recurrent courses of abdominal pain (with or without pancreatitis); the pancreatitis itself, if existed, starts with acute conditions. Main morbidity of the disease is with recurrent episodes of pancreatitis and will lead in some patients to pancreas deficiency or pancreas necrosis ${ }^{3}$. The first manifestation of the disease in our patient was acute necrotizing pancreatitis which was confirmed by diagnostic laparatomy. According to our knowledge, it is the first case that presented with acute necrotizing pancreatitis. 
Hepatosplenomegaly and slight increase in hepatic transaminases are other findings of the syndrome, which arises from fat accumulation in these organs -a phenomenon which was not seen in our patient. Eruptive xanthoma may be caused due to chylomicron phagocytosis by skin macrophages, presenting as yellow deposits over the buttocks and extensor surfaces of the extremities ${ }^{4}$. Lipemia retinalis is another disease manifestation in which retinal vessels have lipaemic look and fundus is seen to be light pink. The deposit may be seen in plasma if the level of triglyceride is more than $4000 \mathrm{mg} / \mathrm{dl}^{5}$. Among other manifestations; dementia, depression, and loss of memory can also be found. Although premature cardiovascular diseases have been identified in a number of the mentioned patients, it does not seem to be a background for premature atherosclerosis ${ }^{6}$.

Apolipoprotein C-II deficiency is a rare genetic defect, which is inherited in an autosomal recessive manner, being rarer than LPL deficiency. Although the clinical findings are similar to LPL deficiency, the symptoms appears later than LPL deficiency. Familial LPL deficiency can be diagnosed by increase in triglyceride level and plasma chylomicron, while Very Low Density Lipoprotein (VLDL) level is low normal. However, there is an increased level of VLDL in familial apo-cll deficiency along with other findings which may be observed in LPL deficiency.Apolipoprotein C-II level is usually assessed by gel electrophoresis. But it was not possible for our patient to evaluate LPL activity and to do an electrophoresis ${ }^{1,4,7}$.

The patients with LPL and apo-cll deficiency can be treated with a fat-restricted diet being nearly $15 \%$ of the total calorie intake. Although fat restriction (10-15 grams daily) is suitable, saturated and non-saturated fat should be limited too, and need for calories should be compensated via medium-chain triglyceride. Furthermore, a greater degree of success was achieved via Omega-3 treatment also ${ }^{8}$. For the patients with apo-cll deficiency (not LPL deficiency) infusion of FFP can be used during an episode of acute pancreatitis. Considering this, we also tried to treat our patient whose disease was in the acute stage of pancreatitis. It led to improvement of the patient's symptoms; during convalescence, the patient was under treatment with omega-3 diet, fat restriction and fat-soluble vitamins.
In conclusion, FCS may be present as acute necrotizing pancreatitis and this rare syndrome should be considered in approaching the patients with acute pancreatitis. In these cases, serum lipids should be completely examined too.

\section{Acknowledgement}

We would like to thank Dr. A.Seyedzadeh for guiding us to write this case report.

\section{References}

1. Mohandas MK, Jemila K, Ajith Krishnan AS et al. Familial chylomicronemia syndrome. Indian J Pediatrics 2005;72:181.

2. Feoli-Fonseca JC, Levy E, Godard M, Lambert M.Familial lipoprotein lipase deficiency in infancy: clinical, biochemical and molecular study. J Pediatr 1998;133:417-423.

3. Lesser PB, Warshaw AL: Diagnosis of pancreatitis masked by hyperlipemia. Ann Intern Med 1975; 82:795-798.

4. Parker F, Bagdade JD, Odland GF, et al: Evidence for the chylomicron origin of lipids accumulating in diabetic eruptive xanthomas: A correlative lipid biochemical, histochemical, and electron microscopic study. J Clin Invest 1970;49:21722187.

5. Thomas VT, Letha S. Lipemia retinalis. Indian J Pediatr 2001;38:925.

6. Benlian $\mathrm{P}$, deGennes $\mathrm{JL}$, Foubert $\mathrm{J}$, et al: Premature atherosclerosis in patients with familial chylomicronemia caused by mutations in the lipoprotein lipase gene. NEngl J Med 1996;335:848854.

7. Shankar KN, Bava HS, Shetty J, Joshi MK. Lipoprotein lipase deficiency. J Postgrad Med 1997;43:81-82.

8. Rouis M, Dugi KA, Previato L, et al: Therapeutic response to medium-chain triglycerides and omega-3 fatty acids in a patient with the familial chylomicronemia syndrome. Arterioscler Thromb Vasc Biol 1997;17:1400-1406. 